\title{
Finite Chainability, \\ Locally Lipschitzian and Uniformly Continuous Functions
}

\author{
G. Marino, G. Lewicki and P. Pietramala ।
}

\begin{abstract}
We present a notion of a finitely chainable subset of a metric space $X$. We show that $Y$ is a finitely chainable subset of $X$ if and only if $f(Y)$ is a bounded subset of $\mathbb{R}$ for any uniformly locally Lipschitzian or uniformly continuous real-valued function $f$ on $X$. As a corollary we reprove the Atsuji theorem in a slightly stronger form.
\end{abstract}

Keywords: Metric spaces, finite chainable subsets, uniformly continuous functions, uniformly locally Lipschitzian functions

AMS subject classification: 26 A 15

\section{Introduction}

In infinite dimensional metric spaces not all continuous images of bounded set's are bounded. Indeed, in 1948 Hewitt [1: p. 69] showed that in a metric space $X$ each continuous, real-valued function is bounded if and only if $X$ is compact.

What happens for uniformly continuous functions? To explain better this problem we begin with

Example 0.1. Let $\left\{e_{n}\right\}_{n \in N}$ be the canonical basis of $l_{2}$ and let $\|\cdot\|$ denote the Euclidean norm. Let $X_{n}$ be the segment joining $e_{n}$ with $e_{n+1}$, i.e. $X_{n}=\left\{e_{n}+t\left(e_{n+1}-\right.\right.$ $\left.\left.e_{n}\right): 0 \leq t \leq 1\right\}$. Let $X=\bigcup_{n=1}^{\infty} X_{n}$. Equip $X$ with two different metrics $\rho$ and $d$ defined by

$$
d(x, y)=\|x-y\|
$$

and

$$
\rho(x, y)= \begin{cases}2^{-n} d(x, y) & \text { if } x, y \in X_{n} \\ 2^{-n} d\left(x, e_{n+1}\right)+D_{n, m}+2^{-m} d\left(e_{m}, y\right) & \text { if } x \in X_{n}, y \in X_{m}(n<m) \\ 2^{-n} d\left(y, e_{n+1}\right)+D_{n, m}+2^{-m} d\left(e_{m}, x\right) & \text { if } y \in X_{n}, x \in X_{m}(n<m)\end{cases}
$$

G. Marino: Univ. of Calabria, Dept. Math., 87036 Arcavacata di Rende, Cosenza, Italy e-mail: gmarino@ccuws4.unical.it

G. Lewicki: Jagiellonian Univ., Dept. Math., 30-059 Kraków, Reymonta 4, Poland e-mail: lewicki@im.uj.edu.pl

P. Pietramala: Univ. of Calabria, Dept. Math., 87036 Arcavacata di Rende, Cosenza, Italy e-mail: papietra@ccuws4.unical.it 
where $D_{n, m}=\sum_{j=n+1}^{m-1} 2^{-j} d\left(e_{j}, e_{j+1}\right)$. Finally, consider a function $f: X \rightarrow \mathbb{R}$ defined by

$$
f(x)=n+t \quad \text { if } x=e_{n}+t\left(e_{n+1}-e_{n}\right) .
$$

Note the following:

a) $(X, d)$ and $(X, \rho)$ are two bounded metric spaces.

b) $d$ and $\rho$ are equivalent but not uniformly equivalent metrics on $X$ (i.e. for every $x \in X$ and $\varepsilon>0$ there exist $\delta_{1}>0$ and $\delta_{2}>0$, depending not only on $\varepsilon$ but also on $x$, such that $\rho(x, y)<\varepsilon$ whenever $d(x, y)<\delta_{1}$ and $d(x, y)<\varepsilon$ whenever $\rho(x, y)<\delta_{2}$.

c) $f$ is a real-valued unbounded function on $X$.

d) $f$ is a uniformly continuous function on the metric space $(X, d)$.

c) $f$ is a continuous but not uniformly continuous function on the metric space $(X, \rho)$.

The situation pointed out in Example 0.1 is not unexpected. Indeed, in 1956 Atsuji [2: Theorem 2] showed that each uniformly continuous real-valued function on a metric space $(X, d)$ is bounded if and only if $X$ is a finite chainable space, i.e. for every $\varepsilon>0$ there are finitely many points $p_{1}, \ldots, p_{l}$ and a positive integer $m$ such that any point of $X$ can be bound with some $p_{j}$ by a finite sequence of $m+1$ points $x=x_{0}, \ldots, x_{m}=p_{j}$ of $X$ satisfying $d\left(x_{k-1}, x_{k}\right)<\varepsilon \quad(k=1, \ldots, m)$.

In this note we introduce and study a notion of a finitely chainable subset of a metric space $X$. The main result of it is Theorem 2.1, which gives a characterization of finitely chainable subsets of $X$. Also, we reprove the Atsuji theorem [2: Theorem 2] in a slightly stronger form.

\section{Finite chainability property}

In the sequel, $X$ denotes a metric space with a metric $d, B(x, r)$ the open ball of a centre $x$ and radius $r$ and $A^{\varepsilon}=\{y \in X$ : dist $(y, A)<\varepsilon\}$ the $\varepsilon$-neighbourhood of a set $A \subset X$. Let $x, y \in X$ and $\varepsilon>0$.

Definition 1.1. An $\varepsilon$-chain of length $m$ joining $x$ with $y$ is a finite sequence of $m+1$ points (not necessarily distinct) of $X, x_{0}=x, \ldots, x_{m}=y$ satisfying $d\left(x_{k}, x_{k-1}\right)<$ $\varepsilon(k=1, \ldots, m)$.

Definition 1.2 (Compare with [2: Definition 3], where the case $Y=X$ has been considered). A subset $Y$ of $X$ is said to be $X$-finitely chainable if for each $\varepsilon>0$ there are a finite set $q_{1}, \ldots, q_{l(\varepsilon)}$ of points of $X$ and a positive integer $m_{Y}=m_{Y}(\varepsilon)$ such that any point of $Y$ can be bound with some $q_{j}(1 \leq j \leq l(\varepsilon))$ by an $\varepsilon$-chain with length $m_{Y}(\varepsilon)$. The function $m_{Y}:[0, \infty] \rightarrow \mathbb{N}, \varepsilon \rightarrow m_{Y}(\varepsilon)$ is said link's number function. It is a non-increasing function.

Example 1.3. We can equip $\mathbb{R}$ with many metrics. For example, the functions

$$
d_{1}(x, y)=|x-y|
$$




$$
\begin{aligned}
& d_{2}(x, y)=\frac{|x-y|}{1+|x-y|} \\
& d_{3}(x, y)=|\arctan (x)-\arctan (y)|
\end{aligned}
$$

are three equivalent metrics on $\mathbb{R}$ but only $d_{1}$ and $d_{2}$ are uniformly equivalent. The following is easy to see:

a) $\left(\mathbb{R}, d_{1}\right)$ is an unbounded and not finite chainable space.

b) $\left(\mathbb{R}, d_{2}\right)$ is a bounded but not finite chainable space.

c) $\left(\mathbb{R}, d_{3}\right)$ is a bounded finite chainable space.

Now we summarize a few properties of $X$-finite chainable subsets.

Proposition 1.4. Let $(X, d)$ be a metric space. Then:

1) The property to be $X$-finite chainable subset is an immersion property, i.e. if $Y$ is $X$-finitely chainable, then $Y$ is $Z$-finitely chainable for every metric space $Z$ which contains metrically $X$.

2) The property to be $X$-finitely chainable is hereditary, i.e. if $Y$ is $X$-finitely chainable, then each subset $Z$ of $Y$ is $X$-finitely chainable.

3) Let $\left\{\left(X_{j}, d_{j}\right), j=1, \ldots, n\right\}$ be a finite family of metric spaces. Then a subset $A=A_{1} \times \ldots \times A_{n}$ in the metric product space $X=\prod_{j=1}^{n} X_{j}$ is $X$-finitely chainable if and only if $A_{j}$ is $X_{j}$-finitely chainable for $j=1, \ldots, n$.

4) Let $\left\{\left(X_{n}, d_{n}\right): n \in \mathbb{N}\right\}$ be a sequence of metric spaces and let $X=\prod_{n=1}^{\infty} X_{n}$ be the Cartesian product of $X_{n}$ endowed with the metric

$$
d\left(\left\{x_{n}\right\},\left\{y_{n}\right\}\right)=\sum_{n=1}^{\infty} 2^{-n} \frac{d_{n}\left(x_{n}, y_{n}\right)}{1+d_{n}\left(x_{n}, y_{n}\right)}
$$

For $A_{n} \subset X_{n} \quad(n \in \mathbb{N})$ consider the set $A=\prod_{n=1}^{\infty} A_{n}$. Then $A$ is $X$-finite chainable if and only if $A_{n}$ is $X_{n}$-finitely chainable for every $n \in \mathbb{N}$. (This is a version of the Tichonoff Theorem for finite chainability.)

5) The property to be $X$-finitely chainable is a metric property but not a topological one, i.e. equivalent but not uniformly equivalent metrics can induce different $X$. chainable subsets. For uniformly equivalent metrics the classes of $X$-finitely chainable subsets with respect to them are the same.

6) The family of $X$-finitely chainable subsets of $X$ contains the family of bounded metrically convex subsets of $X$, whenever $X$ is a complete metric space.

7) The family of $X$-finitely chainable subsets of $X$ is contained (properly in general) in the family of the bounded subsets of $X$.

8) If $E$ is a normed space, then a subset $Y$ of $E$ is $E$-finitely chainable if and only if $Y$ is bounded.

9) Let $Y$ be a subset of a complete metric space $X$. Then $Y$ is relatively compact if and only if $Y$ is $X$-finite chainable and the link's number function admits a maximum.

10) Let $\left(X, d_{X}\right)$ and $\left(Z, d_{Z}\right)$ be iwo metric spaces. Let $f: X \rightarrow Z$ be a uniformly continuous function. Then $f$ maps $X$-finitely chainable subsets of $X$ into $Z$-finitely chainable subsets of $Z$. 
Proof. We only prove statements $4-10$.

Statement 4: Necessity. Let $A=\prod_{n=1}^{\infty} A_{n}$ be $X$-finitely chainable. We show that $A_{n}$ is $X_{n}$-finitely chainable for every $n$. Fix $\varepsilon>0$ and consider $\eta=\frac{\epsilon}{2^{n}(1+\varepsilon)}$. By the $X$-finite chainability of $A$, there exists a number $j(\eta)$ of elements $p^{1}, \ldots, p^{j(\eta)} \in X$ and $m=m(\eta) \in \mathbb{N}$ such that any $x=\left\{x_{n}\right\} \in A$ can be bound with some $p^{i} \quad(1 \leq i \leq j(\eta))$ by an $\eta$-chain in $X x^{0}=x, \ldots, x^{m}=p^{i}$ satisfying $d\left(x^{l-1}, x^{l}\right)<\varepsilon \quad(l=1, \ldots, m)$. Then the $n$-th coordinate $x_{n}$ of $x$ can be bound with the $n$-th coordinate $p_{n}^{i}$ for some $i \in\{1, \ldots, j(\eta)\}$ with an $\varepsilon$-chain in $X_{n}$ of length $m(\eta)$ since

$$
2^{-n} \frac{d_{n}\left(x_{n}^{l-1}, y_{n}^{l}\right)}{1+d_{n}\left(x_{n}^{l-1}, y_{n}^{l}\right)} \leq d\left(x^{l-1}, x^{l}\right)<\eta \quad \text { implies } \quad d_{n}\left(x_{n}^{l-1}, x_{n}^{l}\right)<2^{n} \frac{\eta}{1-2^{n} \eta}=\varepsilon .
$$

Sufficiency. Let $A_{n}$ be $X_{n}$-finitely chainable for every $n$. Take $\varepsilon>0$ and fix $n$ such that $\sum_{k=n+1}^{\infty} 2^{-k}<\frac{\varepsilon}{2}$. Then the thesis follows from property 3 ) applied to $A_{1} \times \ldots \times A_{n}$ and from the fact that

$$
d\left(\left\{x_{k}\right\},\left\{y_{k}\right\}\right)=\sum_{k=0}^{\infty} 2^{-k} \frac{d_{k}\left(x_{k}, y_{k}\right)}{1+d_{k}\left(x_{k}, y_{k}\right)}<\sum_{k=1}^{n} 2^{-k} \frac{d_{k}\left(x_{k}, y_{k}\right)}{1+d_{k}\left(x_{k}, y_{k}\right)}+\frac{\varepsilon}{2} .
$$

Statement 5: Examples 0.1 and 1.3 show that the property to be $X$-finitely chainable is not a topological one. Now let $d_{1}$ and $d_{2}$ be two uniformly equivalent metrics on $X$. Let $A$ be a subset $\left(X, d_{1}\right)$-finitely chainable and let $\varepsilon>0$ be fixed. Then there exists $\eta>0$ such that $d_{1}(x, y)<\eta$ implies $d_{2}(x, y)<\varepsilon$. On the other hand, there are $p_{1}, \ldots, p_{l(\eta)} \in X$ and $m(\eta) \in \mathbb{N}$ such that every $x \in X$ can be bound with some $p_{j}$ by an $\eta$-chain $x=x_{0}, \ldots, x_{m(\eta)}=p_{j}$ such that $d_{1}\left(x_{l}, x_{l+1}\right)<\eta(l=0, \ldots, m(\eta)-1)$. Note that $d_{2}\left(x_{l}, x_{l+1}\right)<\varepsilon$, and consequently $A$ is $\left(X, d_{2}\right)$-finitely chainable.

Statement 6: First of all, a bounded set $A=[0,1] \cup\{2\}$ is $\mathbb{R}$-finitely chainable but not metrically convex. Now, let $A$ be a bounded, metrically convex subset of $X$, i.e. for any $x, y \in A$ there is a point $z \in A$ such that $d(x, y)=d(x, z)+d(y, z)$. A theorem of Menger [3: p. 41] states that a convex and complete metric space contain together with $x$ and $y$ a metric segment whose extremities are $x$ and $y$, that is a subset isometric to an interval of length $d(x, y)$. Hence we see that if $x, y \in A$, there exist $x=x_{0}, \ldots, x_{m}=y$ such that

$$
d(x, y)=\sum_{i=1}^{m} d\left(x_{i-1}, x_{i}\right) \quad \text { and } \quad d\left(x_{i-1}, x_{i}\right)<\varepsilon .
$$

In addition, we can assume that (1.4) holds with

$$
d\left(x_{i+1}, x_{i}\right)+d\left(x_{i+2}, x_{i+1}\right) \geq \varepsilon .
$$

Indeed, since

$$
d\left(x_{i}, x_{i+1}\right)+d\left(x_{i+1}, x_{i+2}\right)=d\left(x_{i}, x_{i+2}\right)
$$

if

$$
d\left(x_{i}, x_{i+1}\right)+d\left(x_{i+1}, x_{i+2}\right)^{\circ}<\varepsilon
$$

we can exclude $x_{i+1}$ from the chain. Hence by (1.4) it follows that $\frac{n \varepsilon}{2} \leq d(x, y)<$ $(n+1) \varepsilon$. Hence, any pair can be bound with an $\varepsilon$-chain of length $m(\varepsilon)<2 \frac{\operatorname{diam}(Y)}{\varepsilon}$. 
Statement 7: Note that Examples 0.1 and 1.3 furnish bounded but not $X$-finitely chainable subsets. The boundedness of an $X$-finite chainable subset $A$ follows from the fact that $A \subset \cup_{j=1}^{m(\varepsilon)} B\left(p_{j}, m_{A}(\varepsilon) \varepsilon\right)$ for fixed $\varepsilon>0$.

Statement 8: Every element $x$ of a bounded set $A \subset E$ can be bound with zero by an $\varepsilon$-chain with knots on the segment $[0, x]$ of length $m(\varepsilon)<\frac{\sup \{\|x\|: x \in A\}}{\varepsilon}$.

Statement 9: For any $\varepsilon>0$ we have $m_{Y}(\varepsilon)=1$. On the other side, let $M=$ $\max \left\{m_{Y}(\varepsilon): \varepsilon>0\right\}$. Fix $\varepsilon>0$. Then there are finite number of points $p_{1}, \ldots, p_{l(\varepsilon / M)} \in$ $X$ such that every point of $Y$ can be bound with some $p_{j}$ by an $\frac{\epsilon}{M}$-chain with length $M$. Thus $Y \subset \cup_{n=1}^{l(\varepsilon / M)} B\left(p_{j}, \varepsilon\right)$.

Statement 10: Fix $\varepsilon>0$. Let $\delta=\delta(\varepsilon)$ be such that $d_{1}(x, y)<\dot{\delta}$,implies $d_{2}(f(x)$, $f(y))<\varepsilon$. Let $Y$ be $X$-finitely chainable subset of $X$. Then there are finite number of points $p_{1}, \ldots, p_{l(\delta)} \in X$ such that any $y \in Y$ can be bound with some $p_{j}$ by a $\delta$-chain of length $m_{Y}(\delta)$. Then any point of $f(Y)$ can be bound with some $f\left(p_{j}\right)$ by an $\varepsilon$-chain of length $m_{Y}(\delta)$

Now we want to examine some properties (frame, amount, length and so on) of the chains with start knots fixed. In this way we will be able to define a non finite chainability measure that will be useful to prove the connexion between $X$-finite chainability, uniform continuity and uniformly local Lipschitz continuity of functions.

Let $(X, d)$ be a metric space and let $\varepsilon>0$ be fixed. We denote by $P(x, \varepsilon, n)$ the set of all points in $X$ which can be bound with $x$ by an $\varepsilon$-chain of length $n$, i.e.

$$
P(x, \varepsilon, n)=\left\{\begin{array}{l|l}
y \in X & \begin{array}{l}
\text { There exist }\left\{z_{1}, \ldots, z_{n-1}\right\} \subset X \text { such that } \\
d\left(x, z_{1}\right)<\varepsilon, d\left(z_{1}, z_{2}\right)<\varepsilon, \ldots, d\left(z_{n-1}, y\right)<\varepsilon
\end{array}
\end{array}\right\}
$$

Moreover, we denote by $P(x, \varepsilon)$ the set of all points in $X$ which can be bound with $x$ by an $\varepsilon$-chain with an arbitrary finite length, i.e.

$$
P(x, \varepsilon)=\bigcup_{n \in \mathbb{N}} P(x, \varepsilon, n) .
$$

With this notation, step by step, it is easy to verify the following

Proposition 1.5.

a) $P(x, \varepsilon, 1)=B(x, \varepsilon)$.

b) $P(x, \varepsilon, n+1)=(P(x, \varepsilon, n))^{e}$ (so any $P(x, \varepsilon, n)$ is an open set). $m \geq n$.

c) $P(x, \varepsilon, n+1)=P(x, \varepsilon, n)$ for some $n$ implies $P(x, \varepsilon, m)=P(x, \varepsilon, n)$ for any

d) $(P(x, \varepsilon))^{e}=P(x, \varepsilon)$, i.e. $P(x, \varepsilon)$ is an isolated set, so if $X$ is a connected metric space, then $P(x, \varepsilon)=X$ for any $x \in X$ and $\varepsilon>0$.

e) $A$ relation $R$ on $X \times X$ defined by $(x, y) \in R$ if and only if $x \in P(y, \varepsilon)$ is an equivalence relation on $X \times X$.

f) The family $\{P(x, \varepsilon): x \in X\}$ is an uniformly isolated partition, i.e. $(P(x, \varepsilon))^{\varepsilon} \cap$ $(P(y, \varepsilon))^{\epsilon}=\emptyset$ if $P(x, \varepsilon) \neq P(y, \varepsilon)$. 
g) $\left(\cup_{i \in I} P\left(x_{i}, \varepsilon\right)\right)^{e}=\cup_{i \in I} P\left(x_{i}, \varepsilon\right)$ for any index set $I$.

h) If there is infinite number of distinct sets $P\left(x_{n}, \varepsilon\right) \quad(n \in \mathbb{N})$ and $(Z, d)$ is an unbounded metric space, then a function $f: X \rightarrow Z$ defined by

$$
f(x)= \begin{cases}0 & \text { if } x \notin \cup_{n \in \mathbf{N}} P\left(x_{n}, \varepsilon\right) \\ z_{n} & \text { if } x \in P\left(x_{n}, \varepsilon\right), n \text { even } \\ w_{n} & \text { if } x \in P\left(x_{n}, \varepsilon\right), n \text { odd }\end{cases}
$$

where $w_{n}, z_{n} \in Z$ are fixed points such that $d\left(w_{n}, z_{n}\right)>n$ is an unbounded uniformly locally Lipschitz function on $X$.

Now, let $Y$ be a bounded subset of $X$. Denote by $N(Y)$ the set of all numbers $\varepsilon>0$ for which $Y$ is chainable by $\varepsilon$-chains with fixed finite length, i.e

$$
N(Y)=\left\{\begin{array}{l|l}
\varepsilon>0 & \begin{array}{l}
\text { There exist } p_{1}, \ldots, p_{i(\varepsilon)} \in X, m_{Y}(\varepsilon) \in \mathbb{N} \\
\text { such that } Y \subset \cup_{j=1}^{i(\varepsilon)} P\left(p_{j}, \varepsilon, m_{Y}(\varepsilon)\right)
\end{array}
\end{array}\right\} .
$$

Of course, if $\varepsilon \in N(Y)$, then the real interval $[\varepsilon, \infty)$ is contained in $N(Y)$. Put

$$
c(Y)=\inf N(Y) \text {. }
$$

This is a measure of non finite chainability of $Y$ and $Y$ is $X$-finitely chainable if and only if $c(Y)=0$.

Moreover, the following is easy to see:

a) $c(Y) \leq \operatorname{diam}(Y)$.

b) $c(A \cup B) \leq \max \{c(A), c(B)\}$.

c) $c(\bar{A})=c(A)$.

d) $A \subset B$ implies $c(A) \leq c(B)$.

e) $X$ is complete if for any decreasing sequence of $X$-finitely chainable closed subsets $\left\{F_{n}\right\}$ one has $\cap_{n \in N} F_{n} \neq \emptyset$.

\section{The main results}

In the sequel $\mathbb{R}$ is endowed with the Euclidean metric. For sake of completeness, we recall that a function $f: X \rightarrow \mathbb{R}$ is said to be uniformly locally Lipschitzian if there are $\rho>0$ and $L>0$ such that

$$
\sup \left\{\frac{|f(x)-f(y)|}{d(x, y)} \mid x, y \in X \text { with } 0<d(x, y)<\rho\right\} \leq L .
$$

Theorem 2.1. Let $(X, d)$ be a metric space and let $Y \subset X$. Then the following conditions are equivalent:

(i) $Y$ is $X$-finitely chainable. 

of. $\mathbb{R}$.

(ii) For any uniformly continuous function $f: X \rightarrow \mathbb{R}, f(Y)$ is a bounded subset

(iii) For any uniformly locally Lipschitzian function $f: X \rightarrow \mathbb{R}, f(Y)$ is a bounded subset of $\mathbb{R}$.

Proof. By Proposition 1.4/8) and 10), (i) implies (ii). Of course, (ii) implies (iii). So we show that (iii) implies (i). Suppose, on the contrary, that $Y \subset X$ is not $X$-finitely chainable. We will construct a real-valued, uniformly locally Lipschitzian function on $X$, unbounded on $Y$. Fix a positive number $\varepsilon_{0}<c(Y)$. Then for any finite set of points $p_{1}, \ldots, p_{l}$ and for any $n \in \mathbb{N}$,

$$
Y \backslash \bigcup_{j=1}^{l} P\left(p_{j}, \varepsilon_{0}, n\right) \neq \emptyset
$$

It can happen or not that there are finitely many points $p_{1}, \ldots, p_{l} \in X$ such that

$$
Y \subset \bigcup_{j=1}^{l} P\left(p_{j}, \varepsilon_{0}\right)
$$

We examine both cases separately.

First case: There exist $p_{1}, \ldots, p_{l}$ such that $Y \subset \bigcup_{j=1}^{l} P\left(p_{j}, \varepsilon_{0}\right)$. Then by (2.2) for some $p_{j}$ we have $P\left(p_{j}, \varepsilon_{0}, n\right) \neq P\left(p_{j}, \varepsilon_{0}\right)$ for any $n \in \mathbb{N}$. Hence, by Proposition $\left.1.5 / \mathrm{c}\right)$; $P\left(p_{j}, \varepsilon_{0}, n\right) \neq P\left(p_{j}, \varepsilon_{0}, m\right)$ for any $n \neq m$. We define $f: X \rightarrow \mathbb{R}$ by

$$
\begin{aligned}
& f(x)= \\
& \begin{cases}0 & \text { if } x \notin P\left(p_{j}, \varepsilon_{0}\right) \\
d\left(x, p_{j}\right) & \text { if } x \in P\left(p_{j}, \varepsilon_{0}, 1\right) \\
(n-1) \varepsilon_{0}+\operatorname{dist}\left(x, P\left(p_{j}, \varepsilon_{0}, n-1\right)\right) & \text { if } x \in P\left(p_{j}, \varepsilon_{0}, n\right) \backslash P\left(p_{j}, \varepsilon_{0}, n-1\right)\end{cases}
\end{aligned}
$$

The function $f$ is unbounded on $Y$ and uniformly locally Lipschitzian on $X \backslash P\left(p_{j}, \varepsilon_{0}\right)$. We show that $f$ is uniformly locally Lipschitzian on $X$. Put $\rho=\varepsilon_{0}$ and fix $x_{1}, x_{2} \in X$ which satisfy

$$
d\left(x_{1}, x_{2}\right)<\rho .
$$

We show that

$$
\left|f\left(x_{1}\right)-f\left(x_{2}\right)\right|<2 d\left(x_{1}, x_{2}\right) .
$$

Since $p_{j}$ and $\varepsilon_{0}$ are fixed, to shorten notation, we will write $P$ instead of $P\left(p_{j}, \varepsilon_{0}\right), P_{n}$ instead of $P\left(p_{j}, \varepsilon_{0}, n\right)$ and $P_{0}=\left\{p_{j}\right\}$. Note that if there is $l \in\{1,2\}$ such that $x_{l} \in P$, then $x_{l} \in P_{n}$ for some $n \in \mathbb{N}$. Put

$$
n_{0}=\min \left\{n \in \mathbb{N}:\left\{x_{1}, x_{2}\right\} \cap P_{n} \neq \emptyset\right\} .
$$

Without loss, we can assume that $x_{1} \in P_{n_{0}}$. By (2.4),

$$
d\left(x_{2}, P_{n_{0}}\right) \leq d\left(x_{1}, x_{2}\right)<\varepsilon_{0} .
$$


Hence $\left\{x_{1}, x_{2}\right\} \subset P_{n_{0}+1}$ by Proposition 1.5/b). Moreover, by (2.6), $\left\{x_{1}, x_{2}\right\} \subset P_{n_{0}+1} \backslash$ $P_{n_{0}-1}$ (if $n_{0}=0,\left\{x_{1}, x_{2}\right\} \subset P_{1}$ ). Note that, if $x_{1}, x_{2} \in P_{n_{0}} \backslash P_{n_{0}-1}$ then, by the definition of $f$,

$$
\left|f\left(x_{1}\right)-f\left(x_{2}\right)\right| \leq\left|d\left(x_{1}, P_{n_{0}-1}\right)-d\left(x_{2}, P_{n_{0}-1}\right)\right| \leq d\left(x_{1}, x_{2}\right) .
$$

Now suppose $x_{2} \in P_{n_{0}+1} \backslash P_{n_{0}}$ and $x_{1} \in P_{n_{0}} \backslash P_{n_{0}-1}$ (hence $n_{0} \geq 1$ ). By Proposition $1.5 / \mathrm{b})$

$$
d\left(x_{2}, P_{n_{0}-1}\right) \geq \varepsilon_{0} .
$$

We show that

$$
\varepsilon_{0}-d\left(x_{1}, x_{2}\right) \leq d\left(x_{1}, P_{n_{0}-1}\right) \leq \varepsilon_{0} .
$$

Since $x_{1} \in P_{n_{0}}, d\left(x_{1}, P_{n_{0}-1}\right)<\varepsilon_{0}$.

Now, suppose on the contrary that

$$
d\left(x_{1}, P_{n_{0}-1}\right)<\varepsilon_{0}-d\left(x_{1}, x_{2}\right) .
$$

Take $y \in P_{n_{0}-1}$ such that $d\left(x_{1}, y\right)<\varepsilon_{0}-d\left(x_{1}, x_{2}\right)$. Then

$$
d\left(x_{2}, P_{n_{0}-1}\right) \leq d\left(x_{2}, y\right) \leq d\left(x_{1}, y\right)+d\left(x_{2}, x_{1}\right)<d\left(x_{2}, x_{1}\right)+\varepsilon_{0}-d\left(x_{2}, x_{1}\right)=\varepsilon_{0},
$$

which is a contradiction with (2.8). Note that in our case

$$
\begin{aligned}
\left|f\left(x_{2}\right)-f\left(x_{1}\right)\right| & =\left|\varepsilon_{0}+d\left(x_{2}, P_{n_{0}}\right)-d\left(x_{1}, P_{n_{0}-1}\right)\right| \\
& =\varepsilon_{0}+d\left(x_{2}, P_{n_{0}}\right)-d\left(x_{1}, P_{n_{0}-1}\right) \\
& \leq \varepsilon_{0}+d\left(x_{2}, P_{n_{0}}\right)-\left(\varepsilon_{0}-d\left(x_{1}, x_{2}\right)\right) \\
& =d\left(x_{2}, P_{n_{0}}\right)+d\left(x_{1}, x_{2}\right) \\
& \leq 2 d\left(x_{1}, x_{2}\right)
\end{aligned}
$$

which proves (2.5) if $\left\{x_{1}, x_{2}\right\} \cap P \neq \emptyset$. Since $f$ is constant on $X \backslash P$, the result is proved.

Second case: For every $p_{1}, \ldots, p_{l} \in X, Y \backslash \cup_{j=1}^{l} P\left(p_{j}, \varepsilon_{0}\right) \neq \emptyset$. By Proposition 1.5/f), there is a sequence $\left\{y_{k}\right\} \subset Y$ such that $P\left(y_{k}, \varepsilon_{0}\right) \neq P\left(y_{h}, \varepsilon_{0}\right)$ for $k \neq h$. Let us define $f: X \rightarrow \mathbb{R}$ by

$$
f(x)= \begin{cases}0 & \text { if } x \notin \cup_{n \in N} P\left(y_{n}, \varepsilon_{0}\right) \\ n & \text { if } x \in P\left(y_{n}, \varepsilon_{0}\right) .\end{cases}
$$

Reasoning as in the case of the previous function, we can show that or $\left\{x_{1}, x_{2}\right\}$ satisfying (2.4) is contained in $X \backslash \cup_{n \in N} P\left(y_{n}, \varepsilon_{0}\right)$ or there exists a fixed $n \in \mathbb{N}$ such that $\left\{x_{1}, x_{2}\right\} \subset$ $P\left(y_{n}, \varepsilon_{0}\right)$. Since $f$ is constant on each $P\left(y_{n}, \varepsilon_{0}\right)$ and on $X \backslash \cup_{n \in N} P\left(y_{n}, \varepsilon_{0}\right),(2.5)$ holds true. The proof is complete

Remark 2.2. We want to give two examples which show that it is necessary to consider two cases examined in the proof of Theorem 2.1:

a) The space $(X, d)$ from Example 0.1 satisfies the first case.

b) $X=\left\{\lambda e_{n}: n \in \mathbb{N}\right.$ and $\left.\lambda \in[1,2]\right\}$, where $e_{n}$ is the canonical basis of $l_{2}$ and $d(x, y)=\|x-y\|_{2}$, satisfies the second case. 
It is worth saying that in Theorem 2.1 we can replace $\mathbb{R}$ with the Euclidean norm by any normed space $(E,\|\cdot\|), E \neq\{0\}$. Indeed, we can define $g: X \rightarrow E$ by $g(x)=f(x) y$ where $y \neq 0$ is a fixed element from $E$ and $f$ is as in the proof of Theorem 2.1.

Corollary 2.3. Let $(X, d)$ be a metric space. Then $X$ is compact if and only if $X$ is finite chainable and each continuous, real-valued function on $X$ is uniformly continuous.

Proof. By Theorem 2.1, any real-valued continuous function is bounded on $X$. Thus the result follows by Hewitt's theorem

Remark 2.4. Metric spaces $(X, d)$ for which any real-valued, continuous function on $X$ is uniformly continuous are widely studied in literature and are known as $U C$ spaces (for references see [4]).

From Theorem 2.1 it is easy to reprove the Atsuji theorem [2: Theorem 2] in a slightly stronger form.

Corollary 2.5 (compare with [2: Theorem 2]). Let $X$ be a metric space. Then $X$ is finitely chainable if and only if $f(X)$ is a bounded subset of $\mathbb{R}$ for any uniformly continuous or uniformly locally Lipschitzian function $f$ on $X$.

\section{References}

[1] Hewitt, E.: Rings of real valued continuous functions. Part I. Trans. Amer. Math. Soc. 64 (1948), 45 - 99.

[2] Atsuji, M.: Uniform continuity of continuous functions of metric spaces. Pac. J. Math. 8 (1958), $11-16$.

[3] Blumenthal, L. M.: Theory and Application of Distance Geometry. Oxford: Clarendon Press 1953.

[4] Beer, G.: UC spaces revisited. Amer. Math. Monthly 95 (1988), 737 - 739. 